\title{
MS42-P08 | LATEST DEVELOPMENTS IN NON-AMBIENT XRD ATTACHMENTS FROM ANTON
}

\section{PAAR}

Puhr, Barbara (Anton Paar GmbH, Graz, AUT); Jones, Andrew O.F. (Anton Paar GmbH, Graz, AUT); Kotnik, Petra (Anton Paar GmbH, Graz, AUT)

Non-ambient XRD has developed into a vital tool for a complete understanding of a materials behavior under different environmental conditions. Parameters such as temperature, pressure, relative humidity, gas environments (including reactive or explosive gases), mechanical load, and electric fields, can be altered allowing samples to be measured in-situ.

This contribution will present the latest developments in non-ambient XRD equipment from Anton Paar which further extend the capabilities of in-house XRD measurements.

These improvements include methods to maximize the temperature accuracy and stability of the non-ambient attachment while minimizing the deviation between the actual sample temperature and that displayed on the nonambient control unit. In addition, new specialized sample holders have been developed for the measurement of battery samples in-operando while changing the sample temperature. Dedicated equipment offers a solution for highly air sensitive samples by means of a sealed transfer to the non-ambient chamber. Furthermore pair distribution function (PDF) measurements are now possible under non-ambient conditions using special PDF parts. Finally, an extended temperature range of the cooling stage for four-circle goniometers could be achieved by improvements of the cooling and heating system.

Presented measurement data prove the functionality of the equipment and point to potential applications. 